\title{
Chapter 7 Using Self-Organizing Maps for Rural Territorial Typology
}

\author{
Marcos Aurélio Santos da Silva \\ Brazilian Agricultural Research Corporation, Embrapa Coastal Tablelands, Brazil \\ Edmar Ramos de Siqueira \\ Brazilian Agricultural Research Corporation, Embrapa Coastal Tablelands, Brazil \\ Olívio Alberto Teixeira \\ Federal University of Sergipe, Department of Economy, Cidade Universitária, Brazil \\ Maria Geovania Lima Manos \\ Brazilian Agricultural Research Corporation, Embrapa Coastal Tablelands, Brazil \\ Antônio Miguel Vieira Monteiro \\ National Institute for Space Research, Image Processing Division, Brazil
}

\begin{abstract}
This work assessed the capacity of the self-organizing map, an unsupervised artificial neural network, to aid the process of territorial design through visualization and clustering methods applied to a multivariate geospatial temporal dataset. The method was applied in the case study of Sergipe's institutional regional partition (Territories of Identity). Results have shown that the proposed method can improve the exploratory spatial-temporal analysis capacity of policy makers that are interested in territorial typology. A new partition for rural planning was elaborated and confirmed the coherence of the Territories of Identity.
\end{abstract}

\section{INTRODUCTION}

Rural territorial typology refers to the classification of commonalities found in contiguous rural areas that may be useful for a well definition of public policies and to discover identity elements. In an integrational perspective, all dimensions must be considered in a spatial analysis. For this study, it was preferred to adopt the definition of territory used by the Ministry of Agrarian Development (MDA, 2005, p.7).

DOI: $10.4018 / 978-1-61692-871-1 . c h 007$

Copyright $($ C 2011, IGI Global. Copying or distributing in print or electronic forms without written permission of IGI Global is prohibited. 
... territories are defined as a physical space, geographically defined, generally continuous, encompassing urban and rural, characterized by multidimensional criteria, such as environment, economy, society, culture, politics and institutions, and a population with relatively distinct social groups that relate internally and externally through specific processes, which can distinguish one or more elements that indicate identity and social, cultural and territorial cohesion.

Territories break down false boundaries (political divisions) among areas and facilitate the process of socio-cultural identity construction, discovery or investigation. The design of public policies also benefits from this strategy, in a way that watersheds, forests, ecosystems and other homogeneous zones can be analyzed altogether. Naturally, there are convergences and divergences about territorial definition, mainly concerning the focus of the study, for example, economical versus environmental analysis. A regional development study implies the use of an interdisciplinary approach, which reveals itself as a very difficult task due to the complexity and uncertainty of this field. Regional studies comprehend constraining factors, such as scales, physical amplitude, quantity and quality of data, diversity of applications, political and practical aspects (Sabourin \& Teixeira, 2002).

The general definition of territory by the MDA (2005) argues that social-cultural-economical cohesion is the main factor to establish a regional aggregation into a territory. Although landscape homogeneity and distribution of natural resources are important aspects, they are not determinant. Therefore, multivariate socio-economical geospatial data integration and interpretation can be useful for policy makers that engage themselves in the process of territory design.

In Brazil, there are many initiatives towards the institutionalization of territories as a new strategy for public management (MDA, 2005; Bandeira, 2006). In this context of interdisciplinary studies and spatial integration, mainly for sustainable de- velopment, rural and urban issues are not viewed as different things, but treated as a systematic problem (Sabourin \& Teixeira, 2002; Flores, 2004; MDA, 2005). Besides, the new trend for self-development by bottom-up as stated by Claval (2008) increases the demand for new and practical approaches to study and generate knowledge for a good territorial partition.

The absence of a territorial theory (Abramovay, 2006) and the rise of territorial policies, at least in Brazil, imply the demand for exploratory methods that could solve practical problems, such as exploratory spatial clustering and, at the same time, it gives us helpful insights for a territorial theory. There are many classical quantitative strategies to perform a multivariate exploratory analysis of geospatial data such as: colored maps, multivariate statistics coupled with these maps, spatial statistics, and geostatistics (Bailey, 1995). In this study, an Artificial Neural Network (ANN) was applied simultaneously for geospatial data visualization and clustering. The objective was to assess the capacity of the ANN, more precisely the Self-Organizing Map (SOM), to find hidden patterns in the geospatial dataset that could be useful for a territorial typology.

This ANN was applied in the analysis of Sergipe's institutional regional partition (Territories of Identity) created by Teixeira et al. (2007) using both subjective and analytical methods. On the other hand, the approach of this work used quantitative geospatial dataset aggregated by municipalities. Results were compared with this official Sergipe's clustering. These territories were chosen because a great effort for Sergipe's territorial partition was performed by academics and officials, so a huge amount of information was gathered and organized. Consequently, the know-how and knowledge about the way the study of regional commonalities is performed increased and, therefore, demanded for integrated quantitative analysis of multisource and multivariated geospatial data. In general terms, the main goal is to establish a semi-automatic territorial typology 
18 more pages are available in the full version of this document, which may be purchased using the "Purchase" button on the product's webpage: www.igi-global.com/chapter/using-self-organizing-maps-rural/48484

This title is available in InfoSci-E-Government, InfoSci-Books, BusinessTechnology-Solution. Recommend this product to your librarian: www.igi-global.com/forms/refer-database-to-librarian. aspx?id=48484

\section{Related Content}

Green Strategic Alignment: Aligning business Strategies with Sustainability Objectives Hui-Ling Wang and Aditya K. Ghose (2011). Handbook of Research on Green ICT: Technology, Business and Social Perspectives (pp. 29-41). www.igi-global.com/chapter/green-strategic-alignment/48417

Mining Geospatial Knowledge on the Social Web

Suradej Intagorn and Kristina Lerman (2011). International Journal of Information Systems for Crisis Response and Management (pp. 33-47). www.igi-global.com/article/mining-geospatial-knowledge-social-web/55306

Application of a Participatory Ex Ante Assessment Model for Environmental Governance and Visualizing Sustainable Redevelopment in Gorj County, Romania Timothy Ehlinger, Lucica Tofan, Mirela Bucur, Jill Enz, Jason Carlson and Richard Shaker (2011). Agricultural and Environmental Informatics, Governance and Management: Emerging Research Applications (pp. 61-86). www.igi-global.com/chapter/application-participatory-ante-assessment-model/54402 Implementing Social Media in Crisis Response Using Knowledge Management Murray E. Jennex (2012). Managing Crises and Disasters with Emerging Technologies: Advancements (pp. 216-228). www.igi-global.com/chapter/implementing-social-media-crisis-response/63314 